Cómo citar este artículo / Referencia normalizada

A Rodríguez Serrano, M Martín-Núñez, S Gil-Soldevila (2017): "Diseño ludológico y realidad aumentada. La experiencia de juego en Pokémon Go! (Niantic, 2016)". Revista Latina de Comunicación Social, 72, pp. 667 a 678. http://www.revistalatinacs.org/072paper/1185/35es.html DOI: 10.4185/RLCS-2017-1185

\title{
Diseño ludológico y realidad aumentada. La experiencia de juego en Pokémon Go! (Niantic, 2016)
}

\section{Ludologic design and augmented reality. The game experience in Pokémon Go! (Niantic, 2016)}

\section{Aarón Rodríguez Serrano [CV] [} https://scholar.google.es/citations?user=93KnFQEAAAAJ\&hl=es] Profesor Investigador Contratado Doctor. Universitat Jaume I (España) serranoa@uji.es

Marta Martín-Núñez [CV] [ - http://orcid.org/0000-0002-9473-1183] [G https://scholar.google.es/citations?user=aOMC9rAAAAAJ\&hl=es] Profesora Ayudante Doctor. Universitat Jaume I (España) mnunez@uji.es

Samuel Gil-Soldevila [CV] [ orcid.org/0000-0002-4711-6268] [G https://scholar.google.es/citations?user=rzMaEj0AAAAJ\&hl=es] Personal Investigador en Prácticas. Universitat Jaume I (España)_ssoldevi@uji.es

\begin{abstract}
s
[ES] Introducción: El objetivo de este trabajo es identificar los procesos narratológicos y ludológicos principales entre los jugadores de Pokémon Go!, el caso más exitoso de realidad aumentada hasta la fecha. Metodología: Para ello, se aplicó una herramienta sociométrica validada por acuerdo inter-jueces a una muestra no probabilística incidental de 332 sujetos. Resultados y conclusiones: Hemos demostrado que no existe correlación entre la experiencia previa narratológica del universo Pokémon con los procesos de juego estrictamente ludoficcionales, sino que la percepción de los jugadores del producto depende más de las mecánicas, reglas y objetivos del propio juego. Esta investigación propone por primera vez un estudio cuantitativo basado en los hábitos de juego de un producto de realidad aumentada y contribuye mediante la propuesta de una herramienta a la medición cuantitativa de percepciones en el proceso ludológico.

[EN] Introduction: The main objective in our research is to identify the narrative and ludological processes between the Pokemon Go! players, the most relevant "augmented reality" video game till now. Methodology: We applied a socio-metric tool validated by different professionals to 332 gamers selected with a non-probabilistic but incidental strategy. Results and conclusions: The results reveal that we can't find a correlation between the previous narrative knowdlege of the Pokémon universe with the perception of the ludofictional processes of the game. The insight of the
\end{abstract}


gamers are more connected with the mechanics, rules and objectives of the game by itself. Our research contributes by the proposal of a new tool for measuring the quantitative inputs of the ludological perceptions. At the same time, we propose for the first time a quantitative research based on the game habits of an augmented reality product.

\section{Keywords}

[ES] Videojuegos; Realidad aumentada; Ludología; Narratología; Pokémon Go!

[EN] Video Games; Augmented Reality; Ludology; Narratology; Pokémon Go!

\section{Contents}

[ES]1. Introducción 2. Método 2.1 Muestra y procedimiento de recogida de datos. 2.2 Instrumento de recogida de información 3. Resultados 3.1 Perfil de los videojugadores. 3.2 Factores narratológicos en la experiencia de Pokémon Go! 3.3. Percepción de las dinámicas de juego. 4. Discusión y conclusiones

[EN] 1. Introduction. 2. Methodology. 2.1 Sample and data collection procedure. 2.2 Data Collection Tool. 3. Results. 3.1 Gamers profile. 3.2 Narratological factors on the Pokémon Go's experience. 3.3 Perception of the game dynamics. 4. Discussion and conclusions

Traducción realizada por Aarón Rodríguez Serrano (Universitat Jaume I - España)

\section{Introducción}

El presente artículo pretende analizar la percepción narratológica y ludológica del videojuego Pokemon Go! (Niantic, 2016) mediante una aproximación cuantitativa. Se trata de un objeto de estudio no exento de problemas, tanto contextuales -el ruido mediático, que ha llegado incluso a problematizar a la comunidad académica (Baranowski, 2016; Serino, Cordrey, McLaughlin, \& Milanaik, 2016)- como estrictamente textuales. Es necesario, por tanto, comenzar realizando una breve puntualización sobre los límites que se encontrarán en nuestra propuesta, así como una serie de rasgos metodológicos previos.

Pokemon Go! es, en el momento de encarar el presente artículo, un texto abierto en tanto sus características estrictamente técnicas y sus patrones ludológicos se encuentran en constante proceso de mutación. La presente reflexión se construye tras una experiencia de juego que atraviesa desde la primera versión hecha pública en España el 15 de Julio de 2016 hasta la versión 0.45.0, activada el 7 de Noviembre del mismo año.

En cierto sentido, esta volatilidad del objeto de estudio no es un rasgo ajeno a la propia naturaleza del videojuego como texto postmoderno (Rodríguez Serrano, 2014). Muy al contrario, la idea misma de utilizar la realidad aumentada como base misma sobre la que levantar el universo ludonarrativo (Azuma, 1997) implica que una parte del software siempre debe permitir un margen de actualización para responder a las realidades geopolíticas del momento. Del mismo modo, como los estudios de José Antonio Planells ya han demostrado (2015), la construcción de mundos virtuales plantea constantes problemas ontológicos que dificultan cualquier labor de análisis que no tome como referencia los sistemas estrictamente ludológicos del mismo. De ahí que nuestra investigación pretenda responder a cuestiones muy acotadas y fundamentadas en dos problemas teóricos con una larga tradición en los game studies: las relaciones entre narratología clásica y mundo específicamente 
ficcional por un lado $-\mathrm{o}$, si se prefiere, la divergencia entre "narrativa estructurada" y "narrativa emergente"-, y la percepción de los elementos ludológicos (reglas, objetivos, ítems, factores de jugabilidad) por parte del jugador o la jugadora.

En el primer caso -las relaciones entre narratología convencional y ludología-, nuestra investigación toma como referencia la ya célebre disputa mantenida entre Murray (1999) y distintos teóricos alineados alrededor de la revista Game Studies (Juul, 1999, 2001) a propósito de la importancia de los elementos narrativos en su relación con la experiencia inmersiva del jugador. Si bien es cierto que en el momento de redactar estas líneas ya se han generado complejos y estimulantes sistemas híbridos (Frasca, 1999, 2003; Pérez Latorre, 2012), hemos creído que la propia naturaleza de Pokémon Go! nos invitaba a preguntarnos por la importancia de la trama, los personajes o sus funciones en relación con la fidelización de cada usuario. No obstante, hay dos novedades reseñables que aporta nuestra investigación frente a los trabajos anteriores: en primer lugar, nuestro material son las percepciones de los propios jugadores a la hora de analizar su propia experiencia. En segundo lugar, nuestro análisis es de orientación cuantitativa y utiliza estadísticos descriptivos, contribuyendo por tanto a completar desde una óptica nueva los anteriores análisis -de corte cualitativo y con predominio del análisis textual lúdico.

En el segundo caso -la percepción de los parámetros ludológicos-, tomamos como referencia dos sistemas complementarios de análisis videolúdico: el trabajo de indicadores propuesto por Navarro Remesal (2016) con su sistemática clasificación de reglas, mecánicas y objetivos y la clasificación de Siabra Fraile (2012) que se vale del segundo Wittgenstein para depurar los universos ficcionales en tres categorías: personajes, items y objetos. Mediante la combinación de ambas propuestas se ha generado una herramienta propia, sometida a rigurosos controles de calidad tal y cómo queda reflejado en el segundo apartado del artículo.

Estas dos áreas de estudio se han sintetizado en cuatro objetivos de investigación que quedan formulados de la siguiente manera:

1. Conocer el perfil concreto de videojugadores o videojugadoras asiduos de Pokémon Go! y sus hábitos básicos de juego: Género, nivel educativo y horas de juego semanales.

2. Determinar la importancia concreta que el conocimiento previo del universo Pokémon y sus características estrictamente narratológicas -ya sea mediante el consumo de la serie o mediante el uso de otros juegos anteriores de la saga- implicaba en su salto a las mecánicas concretas de realidad aumentada.

3. Identificar cómo era la percepción de la experiencia ludológica de los usuarios mediante las herramientas concretas del diseño de juego: objetivos, retos, motivaciones, ítems, carencias detectadas...

4. Explorar las causas concretas del abandono del juego, especialmente por aquellos usuarios que han demostrado o demuestran un notable engagement (o compromiso) con el mismo.

\section{Método}

\subsection{Muestra y procedimiento de recogida de datos}

A la hora de seleccionar la muestra se tuvo en cuenta la necesidad de contar con jugadores especializados que conocieran con precisión las dinámicas de juego. Para localizar específicamente a 
aquellos videojugadores que mostraran mayor implicación hacia la aplicación, se realizó un muestreo no probabilístico incidental en Diciembre de 2016 en el que se seleccionó a la muestra tomando como referencia su actividad verificable en tres comunidades de jugadores diferentes:

1) Grupos de Twitter oficiales y no oficiales específicos de cada comunidad autónoma con mayor número de seguidores.

2) Grupos de Telegram no oficiales con mayor actividad activos en cada comunidad autónoma.

3) Grupos de Facebook no oficiales específicos de cada comunidad autónoma.

El criterio de selección de cada sujeto encuestado exigió un mínimo de tres publicaciones diarias en su espacio personal en redes sociales durante dos semanas de observación directamente relacionadas con el juego (logros, contenidos, noticias externas, concursos...).

Finalmente, se contó con una muestra de 332 sujetos que cumplieron el proceso de selección y que aceptaron tomar parte en la investigación. Para ello, se generó un formulario on-line que respondía al proceso explicitado en el siguiente epígrafe, y se registró en una base de datos cuyo contenido fue tratado mediante el programa SPSS 24.

\subsection{Instrumento de recogida de información}

Al tratarse de una investigación de naturaleza sociométrica, esto es, "fundada sobre una actividad significativa para los sujetos" (Gaitán Moya \& Piñuel Raigada, 2010: 207), optamos por un cuestionario de preguntas cerradas que garantizaba un nivel riguroso de univocidad, pertinencia e importancia siguiendo recientes diseños similares aplicados al ámbito de las nuevas tecnologías y la educación. Para su diseño, tomamos en cuenta las fases prescriptivas que exigen este tipo de herramientas (Alvira, 2011), incluyendo una fase de validación inter-jueces en la que participaron diez doctores (cuatro del área de Comunicación Audiovisual, cuatro del área del diseño de videojuegos, dos del área del diseño metodológico aplicado en ciencias sociales) de cuatro universidades nacionales (dos públicas y dos privadas) con los que se contactó mediante entrevista personal y que también participaron activamente en los procesos de selección de la muestra. Del mismo modo, se realizó una presentación pública de la herramienta en un congreso especializado en febrero de 2017.

Nuestro cuestionario constó finalmente de tres bloques concretos (Tabla 1) seccionados según las diferentes variables y datos que se pretenden estudiar, y que responden a las preguntas de investigación ya explicitadas en el apartado anterior.

El primer bloque (referente al Objetivo de investigación 1) nos permite una breve identificación de la muestra en torno a los dos parámetros clave que dominan los estudios cuantitativos internacionales sobre el consumo de videojuegos: el nivel educativo y el género del videojugador o la videojugadora. Esta diferencia nos permitirá más adelante realizar análisis concretos de contraste de grupos mediante las pertinentes pruebas estadísticas. Del mismo modo, el ítem I.03 incorpora una variable referente al abandono de Pokémon Go! que será de capital importancia en la discusión del Objetivo de Investigación 4. 


\begin{tabular}{|c|c|}
\hline Área & Ítem medido \\
\hline \multirow[t]{3}{*}{$\begin{array}{l}\text { I. Perfil del videojugador o videojugadora } \\
\text { de Pokémon Go! }\end{array}$} & $\begin{array}{l}01 . \text { Nivel educativo del videojugador o la } \\
\text { videojugadora. }\end{array}$ \\
\hline & 02. Género del videojugador o la videojugadora. \\
\hline & 03. Horas de juego de Pokémon Go! semanales. \\
\hline \multirow[t]{3}{*}{$\begin{array}{l}\text { II. Importancia de factores narratológicos } \\
\text { a la hora de jugar a Pokémon Go! }\end{array}$} & $\begin{array}{l}\text { 01. Relación con el mundo narratológico previo } \\
\text { propuesto en la serie de dibujos animados en la que se } \\
\text { basa el videojuego }\end{array}$ \\
\hline & $\begin{array}{l}\text { 02. Relación con los videojuegos anteriores } \\
\text { ambientados en el mundo de Pokèmon. }\end{array}$ \\
\hline & $\begin{array}{l}\text { 03. Valoración de la importancia de la ausencia de } \\
\text { narratividad en las mecánicas que permiten el avance en } \\
\text { Pokèmon Go! }\end{array}$ \\
\hline \multirow{4}{*}{$\begin{array}{l}\text { III. Percepción de las dinámicas de juego } \\
\text { y su relación con el engagement del } \\
\text { usuario. }\end{array}$} & 01. Relación con los objetivos del juego \\
\hline & 02. Relación con los retos del juego \\
\hline & $\begin{array}{l}\text { 03. Relación con las carencias de la experiencia } \\
\text { jugable. }\end{array}$ \\
\hline & 04. Relación con las bonificaciones (items) del juego. \\
\hline
\end{tabular}

El segundo bloque está centrado en los aspectos relacionados con el debate entre narratología y ludología ya explicitado en el epígrafe anterior y correspondiente al Objetivo de Investigación 2. Al tratarse de jugadores experimentados y con un alto compromiso con el mismo, nos parecía necesario medir hasta qué punto dicha aptitud está vinculada tanto al conocimiento previo del universo diegético de Pokémon (Lahti, 2016) -ya sea mediante la familiaridad frente a la serie de dibujos original en el ítem II.01 o de su trama transmedia (Bainbridge, 2014; Geraghty, 2015) en el ítem II.02. Igualmente, en el ítem II.03 exploramos la cuestión clave de conocer hasta qué punto la actividad cotidiana de juego se resentía por no estar anclada en unos parámetros dramáticos y emocionales concretos.

Por último, en el tercer bloque atendemos exclusivamente a las mecánicas de juego para conocer las relaciones entre objetivos, retos, bonificaciones y carencias. Estos cuatro parámetros ofrecen una medición del mundo virtual que permitirá posteriormente, como veremos, entender los parámetros de implicación, así como generar un patrón fiable de los elementos de diseño que funcionan dentro del videojuego y aquellos que no. Serán de utilidad, por lo tanto, para responder a los Objetivos de Investigación 3 y 4.

Debido a la naturaleza de las variables extraídas en el estudio -únicamente atributos, nominales y ordinales- se procederá al análisis de los mismos mediante técnicas propias de la estadística descriptiva. 


\section{Resultados}

\subsection{Perfil de los videojugadores}

El primer bloque arroja una mayor cantidad de usuarios con estudios superiores. Para medir esta variable se utilizó una escala que cubría desde los Cursos inferiores a Bachillerato hasta los estudios universitarios de postgrado concluidos. El grupo estudiado $(\mathrm{N}=332$, DT=1,532) mostró una mayor concentración en videojugadores que se encuentran cursando un grado universitario en la actualidad $\left(\mathrm{F}_{3}=103,31,0 \%\right)$ y en sujetos que ya han terminado sus estudios universitarios de postgrado $\left(\mathrm{F}_{5}=120\right.$, $36,1 \%)$. Del mismo modo, es interesante señalar que se aprecia una concentración nada desdeñable en videojugadores que se encuentran en un nivel escolar anterior a Bachillerato $\left(\mathrm{F}_{1}=73,22 \%\right)$.

En contraste, el género de los participantes sigue siendo indiscutiblemente masculino. En esta ocasión, encontramos un $75,3 \%$ de hombres $(\mathrm{N}=250)$ frente a un $24,7 \%$ de mujeres $(\mathrm{N}=82)$. Si cruzamos ambos datos, el resultado más relevante es sin duda que el mayor número de mujeres jugadoras ha concluido sus estudios de postgrado $(\mathrm{N}=32,39 \%)$, siendo incluso superior a las estudiantes de grado $(\mathrm{N}=23,28 \%)$ y marcando una fuerte diferencia con las jugadoras de bachillerato $(\mathrm{N}=1,1,2 \%)$ y de estudios inferiores $(\mathrm{N}=14,17,1 \%)$.

En cuanto a los hábitos de juego, localizamos un alto nivel de engagement, siendo la moda estadística $(\mathrm{N}=101,30,4 \% \mathrm{DT}=1,277)$ correspondiente a la opción más comprometida: dedicarle más de cinco horas de juego cada semana. Esto queda explicado en parte por la propia selección muestral, pero hay, no obstante, un dato que merece la pena señalar y que posteriormente será utilizado. Pese a tratarse de usuarios que participan activamente en los grupos organizados en torno al juego y que comparten en redes sociales contenidos sobre el mismo de manera cotidiana, encontramos una tasa alta de abandono. Un 18,1\% de los participantes $\left(\mathrm{F}_{5}=60\right)$ afirmó haber dejado de jugar pese a haberse sentido atraído por sus contenidos. Se trata de una cifra inferior a la de aquellos jugadores ocasionales que juegan alrededor de dos horas a la semana $\left(F_{2}=69,20,8 \%\right)$ o alrededor de las cuatro horas $\left(F_{3}=65,19,6 \%\right)$, pero sigue resultando inusualmente alta para la metodología de selección muestral utilizada. Volveremos sobre este dato.

\subsection{Factores narratológicos en la experiencia de Pokémon Go!}

Para intentar medir la importancia que los jugadores atribuían a la parquedad de elementos narratológicos en la construcción del juego se realizó una estrategia doble. En primer lugar, se evaluó la experiencia previa del universo narrativo mediante el consumo de la serie animada Pokémon original (indicador II.1) y, posteriormente, el consumo de otros juegos ambientados en dicho universo y en los que ya se contaba con una propuesta narrativa mucho más compleja (indicador II.2).

En el primer caso, un 75\% ( $\mathrm{N}=249)$ afirmó haber seguido de manera habitual la serie de televisión, y en el segundo, un porcentaje incluso más alto $-76 \%(\mathrm{~N}=251)$ afirmaron haber jugado habitualmente. Todavía es más relevante, al cruzar los datos, comprobar que dentro de la muestra, había una fuerte correlación entre aquellos individuos que habían jugado a alguno de los juegos $y$ habían visto la serie original (un 67,4\% $\mathrm{N}=223$ ), pero también una notable correlación entre aquellos que no habían realizado ninguna de las dos cosas (un $16,6 \% \mathrm{~N}=55$ ). Los datos se encuentran desglosados en la siguiente tabla cruzada: 


\begin{tabular}{|c|c|c|c|c|c|}
\hline & & & Jugar otr & Pokémon & \\
\hline & & & Sí & No & Total \\
\hline Ver serie de TV & Sí & $\mathrm{N}$ & 223 & 26 & 249 \\
\hline & & $\%$ dentro de Ver serie de TV & $89,6 \%$ & $10,4 \%$ & $100,0 \%$ \\
\hline & & $\%$ dentro de Jugar otros Pokémon & $89,2 \%$ & $32,1 \%$ & $75,2 \%$ \\
\hline & & $\%$ del total & $67,4 \%$ & $7,9 \%$ & $75,2 \%$ \\
\hline & No & $\mathrm{N}$ & 27 & 55 & 82 \\
\hline & & $\%$ dentro de Ver serie de TV & $32,9 \%$ & $67,1 \%$ & $100,0 \%$ \\
\hline & & $\%$ dentro de Jugar otros Pokémon & $10,8 \%$ & $67,9 \%$ & $24,8 \%$ \\
\hline & & $\%$ del total & $8,2 \%$ & $16,6 \%$ & $24,8 \%$ \\
\hline Total & & $\mathrm{N}$ & 250 & 81 & 331 \\
\hline & & $\%$ dentro de Ver serie de TV & $75,5 \%$ & $24,5 \%$ & $100,0 \%$ \\
\hline & & $\%$ dentro de Jugar otros Pokémon & $100,0 \%$ & $100,0 \%$ & $100,0 \%$ \\
\hline & & $\%$ del total & $75,5 \%$ & $24,5 \%$ & $100,0 \%$ \\
\hline
\end{tabular}

Al preguntar explícitamente por la importancia que concedían al desarrollo de una trama lineal dentro del juego (Indicador II.03), encontramos resultados sensiblemente diferentes. Un 39,2\% del todo $(\mathrm{N}=130)$ consideraba problemático que Pokémon Go! no contara con un desarrollo lineal, mientras que un 60,8\% (N=202) afirmó no sentirse especialmente preocupado por ello.

Ahora bien, en una segunda fase del análisis de datos hemos advertido gracias a la prueba de $\chi^{2}$ que no se puede establecer con seguridad una correlación entre los primeros indicadores (II.01 y II.02) con el tercero (II.O3). Esto implica que no podemos concluir ni que los consumidores habituales de la serie $\left(\mathrm{p}<0.05, \chi^{2}=0.37\right)$ ni que los jugadores de anteriores entregas $\left(\mathrm{p}<0.05, \chi^{2}=0.22\right)$ detecten invariablemente como un problema la falta de narratividad en el desarrollo del juego.

\subsection{Percepción de las dinámicas de juego}

El último tramo estaba organizado en torno a la percepción sobre su objetivo principal como videojugador, los motivos más importantes para seguir jugando, las bonificaciones y las carencias de la experiencia jugable. A continuación ofrecemos el desglose concreto de todos los resultados recogidos: 


\begin{tabular}{|c|c|c|c|}
\hline \multicolumn{2}{|l|}{ Área } & Porcentaje & $\mathrm{N}$ \\
\hline \multirow{4}{*}{$\begin{array}{l}\text { Objetivos } \\
(\mathrm{DT}=0,822)\end{array}$} & Capturar nuevos pokémon & 83,7 & 278 \\
\hline & Pelear contra equipos rivales & 8,4 & 28 \\
\hline & Entrenar en mi gimnasio & 0,3 & 1 \\
\hline & Hacer evolucionar pokémons ya cazados & 7,5 & 25 \\
\hline \multirow{4}{*}{$\begin{array}{l}\text { Retos } \\
(\mathrm{DT}=0,88)\end{array}$} & Encontrar pokémons nuevos & 74,1 & 246 \\
\hline & Subir de nivel pokémons ya capturados & 9,9 & 33 \\
\hline & Subir de nivel al personaje principal & 10,5 & 35 \\
\hline & Conseguir logros nuevos & 5,4 & 18 \\
\hline \multirow{7}{*}{$\begin{array}{l}\text { Carencias del juego } \\
(\mathrm{DT}=2,013)\end{array}$} & Los jugadores deberían poder intercambiar pokémons & 28 & 93 \\
\hline & Los jugadores deberían poder enfrentarse entre ellos & 18,1 & 60 \\
\hline & El avatar debería ser más personalizable & 2,1 & 7 \\
\hline & Debería ser más fácil encontrar pokémons nuevos & 18,1 & 60 \\
\hline & El sistema de lucha debería ser más completo & 12,3 & 41 \\
\hline & Debería haber más variedad de pokémons para cazar & 17,5 & 58 \\
\hline & Debería haber mayor variedad de recompensas & 3,9 & 13 \\
\hline \multirow{5}{*}{$\begin{array}{l}\text { Recompensa más útil } \\
(\mathrm{DT}=1,37)\end{array}$} & Pokéballs ultra & 10,2 & 34 \\
\hline & Incienso & 5,4 & 18 \\
\hline & El huevo de la suerte & 21,7 & 72 \\
\hline & Los módulos cebo & 8,4 & 28 \\
\hline & Incubadoras & 54,2 & 180 \\
\hline
\end{tabular}

En principio, podemos observar que los objetivos y los retos parecen ofrecer resultados más homogéneos, con una desviación típica inferior a 1 en ambos casos. Del mismo modo, la concentración de datos apunta con claridad en ambos indicadores a la captura de nuevos pokémons (III. $0183,7 \%, \mathrm{~N}=278$ y III.02 74,1\%, N=246) como el motor principal sobre el que los jugadores basan la experiencia. En el caso de las carencias del programa, resulta arriesgado ofrecer datos concluyentes debido a la dispersión muestral, si bien es cierto que se encuentra una cierta concentración en los ítems relacionados con la interacción de jugadores $\left(\mathrm{F}_{1} 28 \%, \mathrm{~N}=93\right.$ y $\mathrm{F}_{2} 18,1 \%$, $\mathrm{N}=60)$, así como en aquellos que -de nuevo- hacen referencia a la captura de pokémons $\left(\mathrm{F}_{4} 18,1 \%\right.$, $\mathrm{N}=60$ y $\left.\mathrm{F}_{6} 17,5 \%, \mathrm{~N}=58\right)$. Por último, en el apartado de recompensas, se nota una gran concentración en dos ítems: los huevos de la suerte -utilizados principalmente para permitir al personaje subir de nivel- $\left(\mathrm{F}_{3} 21,7 \%, \mathrm{~N}=72\right)$ y sobre todo, las incubadoras -que amplían la 
posibilidad de cazar pokémons extraños, de otras zonas geográficas o de otras áreas lejanas al jugador $\left(\mathrm{F}_{5} 54,2 \%, \mathrm{~N}=180\right)$.

\section{Discusión y conclusiones}

Una vez llegados a este punto, ya podemos realizar un análisis más complejo de los indicadores obtenidos. Para ello, seguiremos la disposición de los objetivos de investigación planteados.

En cuanto al perfil del videojugador (Objetivo I), podemos observar que la gran mayoría de participantes sigue siendo masculino, lo que confirma las conclusiones de otros estudios desarrollados en el mismo ámbito (Aierbe-Barandiaran \& Oregui-González, 2016; Chicharro Merayo, 2014) remarcando la desigualdad todavía existente en el campo estudiado en los hábitos de consumo y preferencias (Coyne, Jensen, Smith, \& Erickson, 2016; Ricoy \& Ameneiros, 2016). Del mismo modo, al tratarse de un juego únicamente disponible en terminales móviles, nuestros resultados confirman también los trabajos que apuntan a una mayor dispersión en la franja de edad de los jugadores, así como el acceso cada vez más temprano a contenidos lúdicos en esos terminales (Bond, 2013; Madhuri, Olsen, Sigsgaard, \& Kheifets, 2016).

En cuanto al segundo objetivo, referido a los parámetros narratológicos, parece también bastante claro que el jugador de Pokémon go! ha contado con una experiencia previa de las líneas temáticas del universo si bien -y este dato nos parece crucial-, toda esa experiencia no ha sido suficiente para fidelizar a los usuarios más allá del contacto inicial con el juego. Con el ya citado fracaso de la prueba $\chi^{2}$, parece evidente que lo que atraía a los jugadores no era la posibilidad de "actualizar narrativamente" la historia de Ash llevándola a una nueva plataforma, sino por el contrario, el propio funcionamiento de mecánicas de realidad aumentada aplicadas a una serie de objetivos mediante un terminal móvil. Esto pondría en duda las teorías sobre el carácter necesariamente nostálgico de Pokémon go!, separándolo de otro tipos de consumo de videojuegos retro (Márquez, 2012; Planells, 2013).

El tercer objetivo apunta con claridad a la dinámica principal que valoraban la inmensa mayoría de los jugadores: la captura de nuevos pokémons está muy por encima de las acciones de combate, entrenamiento, aumento de nivel u obtención de logros "reglados" por el propio software. Esto, evidentemente, implica que la propia vida del juego queda limitada dramáticamente por sus propias mecánicas. Con anterioridad a la actualización de Diciembre de 2016, el juego únicamente permitía la captura de alrededor de 150 tipos diferentes, de los cuales varios se circunscribían a zonas geográficas concretas. Pese a que el juego prometía la diversidad de ejemplares disponibles -la llamada "rotación de nidos"-, se puede confirmar que el principal problema a la hora de mantener la jugabilidad está relacionado con un desequilibrio entre las acciones de juego más valoradas -captura (III.01 y III.02)-, la percepción de carencias por parte de los jugadores -falta de nuevos ejemplares (III.03) y los ítems destinados a la consecución de dicha acción -incubadoras (III.04).

Esto queda, a su vez, confirmado en el cuarto objetivo de investigación, destinado a localizar los errores en el diseño de juego que han generado un mayor índice de abandono. Para ello, es necesario analizar las percepciones sobre su experiencia de aquellos jugadores que ya han abandonado. El resultado queda expuesto en la siguiente tabla, en la que reseñamos la moda estadística en los diferentes indicadores: 


\begin{tabular}{|c|c|c|c|}
\hline Categoría (Indicador) & Valor correspondiente a la moda estadística & Porcentaje & $\mathrm{N}$ \\
\hline Objetivo & $\mathrm{F}_{1}$ : Encontrar nuevos Pokémons & $75 \%$ & 45 \\
\hline Reto & $\mathrm{F}_{1}$ : Encontrar nuevos Pokémons & $86,7 \%$ & 52 \\
\hline $\begin{array}{l}\text { Carencia del diseño de } \\
\text { juego }\end{array}$ & $\begin{array}{l}\mathrm{F}_{1} \text { : Deberían poder intercambiarse Pokémons entre } \\
\text { jugadores }\end{array}$ & $28,3 \%$ & 17 \\
\hline Recompensa & $\mathrm{F}_{3}$ : Incubadoras & $33 \%$ & 20 \\
\hline
\end{tabular}

Como se puede apreciar, la concentración de altos porcentajes en los dos primeros indicadores desvela claramente que los jugadores no han encontrado en el juego una continuidad basada en la caza de nuevos pokémons que les motivara lo suficiente como para continuar participando.

En resumen, hemos conseguido trazar con cierta precisión una fotografía coherente del proceso ludoficcional sobre el que se asienta Pokémon go!: una propuesta capaz de ser disfrutada por jugadores de todas las edades gracias a la sencillez de sus mecánicas iniciales, pero incapaz de mantener esa atención por la falta de valor atribuido a las acciones secundarias -especialmente a las de entrenamiento y enfrentamiento-, a la ausencia de dinámicas de interactuación con otros jugadores humanos y por el diseño insuficiente de los ítems y los logros que deberían haber servido para aumentar la experiencia de juego.

Lógicamente, la presente investigación podrá ser completada con nuevos estudios en el campo de la ludoficción aplicada a la realidad aumentada, ya sea a través de las futuras ampliaciones de Pokémon go! o mediante la comparativa con futuros juegos desarrollados bajo estos mismos parámetros. No obstante, creemos que la herramienta desarrollada permitirá valorar, de manera sencilla y ordenada, las variables narratológicas y ludológicas de los juegos portátiles más sencillos, por lo que esperamos seguir refinándola y ampliándola en el futuro.

- Este artículo ha sido posible gracias a la financiación del Ministerio de Economía y Competitividad para el subproyecto I+D "El sistema de investigación sobre prácticas sociales en Comunicación: mapa de proyectos, grupos, líneas, objetos de estudio y métodos" (CSO2013-47933-C4-4-P), y de la Universitat Jaume I en el marco del proyecto "La crisis de lo real: la representación documental e informativa en el entorno de la crisis financiera global” (P1·1A2014-05), ambos proyectos bajo la dirección de Javier Marzal Felici.

\section{Bibliografía}

Aierbe-Barandiaran, A., \& Oregui-González, E. (2016). Valores y emociones en narraciones audiovisuales de ficción infantil. Values and Emotions in Children's

Audiovisual Fictional Narratives., 24(47), 69-77. http://doi.org/10.2337/dc10 S011

Alvira, F. (2011). La encuesta: una perspectiva general metodológica. Colección de Cuadernos Metodológicos Num 35, 6. 
Azuma, R. (1997). A survey of augmented reality. Presence: Teleoperators and Virtual Environments, 6(4), 355-385. http://doi.org/10.1.1.30.4999

Bainbridge, J. (2014). It's a Pokemon World: The Pokemon franchise and the environment. International Journal of Cultural Studies, 17, 399-414. http://doi.org/10.1177/1367877913501240

Baranowski, T. (2016). Pokémon Go, go, go, gone? Games for Health Journal. http://doi.org/10.1089/g4h.2016.01055.tbp

Bond, E. (2013). Mobile phones, risk and responsability: Understanding children's perceptions. Cyberpsychology: Journal of Psychological Research on Cyberspace, 7(1).

Chicharro Merayo, M. (2014). Jóvenes, ficción televisiva y videojuegos: espectáculo, tensión y entrenimiento. Tendencias generales de consumo. Revista de Estudios de Juventud, 106(77-91).

Coyne, S. M., Jensen, A. C., Smith, N. J., \& Erickson, D. H. (2016). Super Mario brothers and sisters: Associations between coplaying video games and sibling conflict and affection. Journal of Adolescence, 47, 48-59. http://doi.org/10.1016/j.adolescence.2015.12.001

Frasca, G. (1999). Ludology meets narratology: Similitude and differences between (video) games and narrative. Ludology. Org, 1-15.

Frasca, G. (2003). Ludologists Love Stories, Too : Notes From a Debate That Never Took Place. Game Studies, 92-99. http://doi.org/10.1007/s10055-009-0124-3

Gairín Sallán, J. (2014). Estudio sobre los usos y abusos de las Tecnologías de la Información y la Comunicación en adolescentes. No 135.

Gaitán Moya, J., \& Piñuel Raigada, J. L. (2010). Técnicas de investigación en comunicación social: Elaboración y registro de datos. Madrid: Síntesis.

Geraghty, L. (2015). An evolutionary journey: Pokémon, mythic quests and the culture of challenge. In K. Beeler \& S. Beeler (Eds.), Children's Films in Digital Age. Essays on audience, adaptation and consumer culture (pp. 78-88). Jefferson: McFarland \& Company.

Juul, J. (1999). A Clash between Game and Narrative. Danish Literature, 1, 95.

Juul, J. (2001). Games Telling stories? - A Brief Note on Games and Narratives. Game Studies, 1(1), $1-12$.

Lahti, I. (2016). Representation of Primary Characters in Narrative-based Games. DiVA (Digitala Vetenskapliga Arkivet), 1, 1-32.

López Gómez, J. E., García García, F. \& García Guardia, M. L. (2012). La capacidad de constancia es un componente básico de la competencia afán de logro: orientación a resultados en alumnos de educación secundaria.Étic@Net, 1(12), 38-65.

Madhuri, S., Olsen, J., Sigsgaard, T., \& Kheifets, L. (2016). Trends in cell phone use among children in the Danish national birth cohort at ages 7 and 11 years. Journal of Exposure Science \& Enviromental Epidemiology, 26, 606-612.

Márquez, I. V. (2012). Nostalgia videolúdica: un acercamiento al movimiento chiptune. Trans. Revista Transcultural de Música, (16), 1-21. 
Murray, J. H. (1999). Hamlet en la holocubierta : el futuro de la narrativa en el ciberespacio. Barcelona: Paidós.

Navarro Remesal, V. (2016). Libertad dirigida: Una gramática del análisis y diseño de videojuegos. Santander: Shangrila.

Pérez Latorre, Ó. (2012). El Lenguaje videolúdico : análisis de la significación del videojuego. Barcelona : Lartes.

Planells, A. J. (2013). Arte pixelado, nostalgia y géneros perdidos: "Resonance" o el resurgir de la aventura gráfica clásica. Arte, DIseño e Ingeniería, 2, 8-15.

Planells, A. J. (2015). Videojuegos y mundos de ficción. Madrid: Cátedra.

Ricoy, M. del C., \& Ameneiros, A. (2016). Preferencias, dedicación y problemáticas generadas por los videojuegos: Una perspectiva de género. Revista Complutense de Educación, 27(3), 1291-1308. http://doi.org/10.5209/rev_RCED.2016.v27.n3.48445

Rodríguez Serrano, A. (2014). Cuando los videojuegos escribieron el Holocausto: Análisis de Wolfenstein: The New Order(Machinegames, 2014). Historia Y Comunicación Social, 19, 193-207. http://doi.org/10.5209/rev_HICS.2014.v19.47292

Serino, M., Cordrey, K., McLaughlin, L., \& Milanaik, R. L. (2016). Pokémon Go and augmented virtual reality games: a cautionary commentary for parents and pediatricians. Current Opinion in Pediatrics, 1-5. http://doi.org/10.1097/MOP.0000000000000409

Siabra Fraile, J. A. (2012). Bosquejo de una metafísica del videojuego. El Ejido: Círculo Rojo.

Torrado Fonseca, M. (2004). Estudios de encuesta. Metodología de la Investigación Educativa, 231257.

\section{Cómo citar este artículo / Referencia normalizada}

A Rodríguez Serrano, M Martín-Núñez, S Gil-Soldevila (2017): "Diseño ludológico y realidad aumentada. La experiencia de juego en Pokémon Go! (Niantic, 2016)". Revista Latina de Comunicación Social, 72, pp. 667 a 678.

http://www.revistalatinacs.org/072paper/1185/35es.html

DOI: $\underline{10.4185 / R L C S-2017-1185}$

- En el interior de un texto:

... A Rodríguez Serrano, M Martín-Núñez, S Gil-Soldevila (2017: 667 a 678)...

o

... A Rodríguez Serrano et al, 2017 (667 a 678)...

Artículo recibido el 18 de abril. Aceptado el 17 de junio. Publicado el 20 de junio de 2017 